\title{
Phase Structures of Magnetic Impurity Models with Two-Body Hybridization
}

\author{
Yue Yu and Wan-Peng Tan \\ Institute of Theoretical Physics, Academia Sinica, Beijing 100080, China
}

\begin{abstract}
The most general model with a magnetic impurity coupled to hybridizing and screening channels of a conduction band is considered. The partition function of the system is asymptotically equivalent to that of the multi-component kink plasma with a weak external field. The scaling properties of the models for finite $U$ are sketched by using the Anderson-Yuval-Hamann-Cardy poor man's scaling theory. We point out that it is proper to include a two-body hybridization in order to obtain correct renormalization flows. The phase structures are studied graphically for the general model and various reduced models. A Fermi-non-Fermi liquid phase transition is found for all the models. We also show all possible phases with different finite temperature behaviors though they have the same Fermi liquid fixed point at low temperature. We also discuss the fixed point behaviors in the mixed valence state regime.
\end{abstract}

\section{INTRODUCTION}

The normal-state properties of the Cu-O-based materials had been understood phenomenologically on the basis of the existence of the non-Fermi-liquid(NFL)-like groundstates [1] [2]. In one dimension (1-d), it is wellknown that the Luttinger liquid states appear universally in a wide class of interacting systems [3]. The NFL behaviors are shown for arbitrarily weak interactions in 1-d. However, for the dimensions higher than one, the perturbative renormalization group analysis does not support the NFL behaviors [4]. This promotes the non-perturbative approaches to be developed. In 1-d, bosonization is a powerful non-perturbative method [5]. For higher dimensions, several ways are applied. People try to generalize the 1-d bosonization procedure to higher dimensional one. Haldane [6], Houghton and Marston [7] provided a kind of bosonization procedures in higher dimensions. More recently, many authors use this kind of procedure to discuss whether the NFL behaviors appear in higher dimensions [8]. The other subject that many investigations focus on is the confinement of the Luttinger liquid behavior among the weakly interacting 1-d Tomonaga-Luttinger chains [9]. There still are some arguments for this subject 10. The way related to this paper is to study such models that the properties of the models can be obtained either exactly or with the welldefined limit of validity. For example, various single impurity models are good objects to be studied. Research for them can be thought of as a starting point to do the corresponding lattice models related to the physics of the high temperature superconduction materials.

It has been known that at low energies which are much less than the Kondo temperature, the groundstates of simple magnetic impurity models 111$]$ [12] are Fermi liquid (FL) [13] [14]. It has recently been argued that the generalization of those models is closely related to the physics of the $\mathrm{Cu}-\mathrm{O}$-based materials [15] [16] 17] [18]. It is important that a phase transition between FL and NFL states has been found in investigations of single impurity models because the NFL behavior can explain many anomalies of the normal state of high temperature super- conductors [1] [2].

The Kondo model is one of typical impurity models. Through the bosonization or the partition function analog, the Kondo problem can be mapped to a one-channel resonant-level model and has been shown that the ground state for the energy well below the Kondo temperature is of the FL behavior 19]. The multi-channel generalized model considers all of conduction channels interact with the impurity orbital but only one channel hybridizes with the impurity orbital. Following the terminology used by the authors of [15] [16] we call later the 0-channel or the hybridizing channel and others the screening channels. For the finite range interactions a FL-NFL phase transition has been shown by multiplicative renormalization group 20] in 16] and by the poor man's scaling theory 21] in [17. Physically, as pointed out by Zhang, Yu and Su [17], the phase transition is caused by a competition between two different kinds of metallic behaviors, the NFL behavior of the multi-channel X-ray edge problem 22 in the screening channels and the FL behavior of the one-channel Kondo problem in the hybridizing channel. A more general impurity model is the Anderson model [11] [12]. Perakis et al 15] studied the multi-channel Anderson model and also found that there are NFL phases by using Wilson's numerical renormalization group method [13]. A similar case also occurs for a generalized Hubbard model in infinite dimensions [18].

In a previous Brief Report [23] by one of us, cooperating with Li and d'Amberumenil, one showed that it is necessary to introduce a hybridization of two hybridizing electrons with opposite spin hopping onto impurity orbital in order to keep the renormalization group's transitivity in the multi-channel models. Here, we would like to give our full investigation to this issue. We will generalize the approach used in the particle-hole $(\mathrm{PH})$ symmetric model to more general magnetic impurity models in the spirit of the Anderson-Yuval-Hamann-Cardy (AYHC) poor man's scaling theory [21] 24], which has been generalized to a form with symmetry breaking [25]. We also find that in the most general model for finite $U$ a fully renormalizable model must include a hybridization of two hybridizing electrons with opposite spin hop- 
ping onto impurity orbital. Because we try to describe the low energy behaviors under a low electron density in the conduction band, we apply the bosonization procedure for impurity models [26]. The Hilbert space of the system can be divided into four sub-spaces characterized by the impurity states. The 'dipole' operators between sub-spaces are of their transitivity. This transitivity leads to an asymptotic equivalence, via bosonization, between the general model and a multi-component kink plasma model. This has been shown by Si and Kotliar in the infinite $U$ case [18,25]. On the other hand, according to the transitivity, the 'charges' of the plasma are shown to obey the Jacobi identities, which implies that the 'charges' form an adjoint representation of some Lie algebra. The Lie algebra is shown to be solvable. Under the PH symmetry, the dimensions of the Lie algebra are reduced, which is closely related to the changes of the phase structures. The phase structures of various models can be recognized from the corresponding renormalization group equations which are derived by using the AYHC poor man's scaling theory and its symmetry breaking generalization. The phase diagrams for the models may be drawn systematically by Cardy's graphical method [24]. For each model, we find that the phase in which all fugacities of the plasma are irrelevant is the NFL phase. As long as one of the fugacities varies from an irrelevant to a relevant one, the FL-NFL phase transition occurs. For the irrelevant phase, the model at fixed point is reduced to the X-ray edge problem, whose fixed point behaviors are controlled by the NFL. A competition between those two different type metallic states causes the FL-NFL phase transition. Cardy's graphical method also shows that it is possible that there are phase transitions at finite temperature between other phases although they have the same low temperature Fermi liquid fixed point. However, the renormalization group equations may not be valid quantitatively in the strongly coupling regions. We also study fixed point behaviors in the mixed valence regime. One sees that there are four kinds of the different fixed points. One is in the strong coupling limit corresponding to the FL one and another is in the weak coupling limit which is the NFL one. We calculate various single-particle and two-particle correlation functions in the weak coupling limit. Other two are the intermediate coupling regimes in which there are local NFL behaviors although the infrared orthogonality catastrophe of the X-ray edge type does not appear. Comparing to the results of the infinite $U$ model [25], we have one more intermediate mixed valence regime which is related to the two-body hybridization irrelevant.

This paper is organized as follows. In section II, we define the general model under the consideration and explain various reduced models. In section III, the partition function of the theory is expressed by the histories of the impurity states and is mapped into that of a multi-component kink plasma. We also briefly review the bosonization procedure for the problem. In section IV, we study the phase structures of the theory. In sec- tion $\mathrm{V}$, the fixed point behaviors of the mixed valence states are discussed. The last section gives our conclusion. The appendices talk about the Lie algebra related to the 'charges' of the kinks and give some comments on the derivation of the renormalization group equations.

\section{SINGLE IMPURITY MODELS WITH TWO-BODY HYBRIDIZATION}

We begin with the derivation of the general model. Assume a single impurity atom is located at the origin and has a localized electron orbital $\phi_{d}(\mathbf{r})$ with the local impurity level $\epsilon_{d \sigma}$. Other electrons are in the conduction band described by the wave function $\phi_{\mathbf{k}}(\mathbf{r})$ and energy $\epsilon_{\mathbf{k}}$. A general state function is the summation over all possible states,

$$
\psi(\mathbf{r})=\sum_{\mathbf{k} \sigma} \phi_{\mathbf{k}}(\mathbf{r}) X_{\sigma} c_{\mathbf{k} \sigma}+\sum_{\sigma} \phi_{d}(\mathbf{r}) X_{d \sigma} d_{\sigma},
$$

where $X_{\sigma}$ and $X_{d \sigma}$ are the spin wave functions and $\sigma$ labels spin up $\uparrow$ and down $\downarrow$. More generally, we consider the spin wave function of impurity, $X_{d \sigma}$ as an operator $X_{d \sigma}=x_{\sigma}\left(1-n_{d}\right)+\tilde{x}_{\sigma} n_{d}$, which leads to the different wave functions for the empty, single occupation and double occupation of the impurity orbital. Under this consideration, the Fano-Anderson model is generalized as

$$
\begin{aligned}
H_{G F A} & =\sum_{\mathbf{k}, \sigma} \epsilon_{\mathbf{k}} c_{\mathbf{k} \sigma}^{\dagger} c_{\mathbf{k} \sigma}+\sum_{\sigma} \epsilon_{d \sigma} d_{\sigma}^{\dagger} d_{\sigma} \\
& +\frac{1}{\sqrt{L}} \sum_{\mathbf{k}, \sigma}\left[\left(t_{\mathbf{k} \sigma}\left(1-n_{d}\right)+\tilde{t}_{\mathbf{k} \sigma} n_{d}\right) c_{\mathbf{k} \sigma}^{\dagger} d_{\sigma}+\text { h.c. }\right]
\end{aligned}
$$

where $\epsilon_{d \sigma}$ is the impurity level which is taken as the spin independence, $\epsilon_{d}$, without losing the generality. Assume only the conduction electrons in the 0-channel hybridize with the impurity orbital and the hybridizations are $k$ independent in the model. The chemical potential is set to zero but the Hamiltonian (2.2) is still particle-holeasymmetry.

To discuss the interesting physical phenomenon, the electron-electron interaction must be introduced. We consider only the finite range interaction. Besides the Hubbard-type interaction in the Anderson model we also keep consideration to other two types of interactions, the spin-exchange interaction and a two-body hybridization which describes the two hybridizing electrons with opposite spins hopping onto the impurity orbital simultaneously. By using the wave function (2.1), the model Hamiltonian reads

$$
\begin{aligned}
H & =\sum_{k>0 ; \sigma, l} \epsilon_{k} c_{k \sigma l}^{\dagger} c_{k \sigma l}+\epsilon_{d} n_{d}+U n_{d \uparrow} n_{d \downarrow} \\
& +\frac{1}{\sqrt{L}} \sum_{\sigma}\left[\left(t_{1 \sigma}\left(1-n_{d}\right)+\tilde{t}_{1 \sigma} n_{d}\right) c_{\sigma 0}^{\dagger} d_{\sigma}+h . c .\right]
\end{aligned}
$$




$$
\begin{aligned}
& +\frac{t_{2}}{L} \sum_{\sigma}\left(c_{\sigma 0}^{\dagger} c_{-\sigma 0}^{\dagger} d_{\sigma} d_{-\sigma}+h . c .\right) \\
& +\frac{1}{L} \sum_{\sigma l}\left[V_{x \sigma l}\left(2-n_{d}\right)+\tilde{V}_{x \sigma l}\left(n_{d}-1\right)\right] c_{\sigma l}^{\dagger} c_{\sigma l} d_{\sigma}^{\dagger} d_{\sigma} \\
& +\frac{1}{L} \sum_{\sigma l} V_{y l} c_{\sigma l}^{\dagger} c_{-\sigma l} d_{-\sigma}^{\dagger} d_{\sigma} \\
& +\frac{1}{L} \sum_{\sigma \sigma^{\prime} l}\left[V_{l \sigma}\left(2-n_{d}\right)+\tilde{V}_{l \sigma}\left(n_{d}-1\right)\right] c_{\sigma l}^{\dagger} c_{\sigma l} d_{\sigma^{\prime}}^{\dagger} d_{\sigma^{\prime}}
\end{aligned}
$$

The first four terms in (2.3) are the generalized FanoAnderson model (2.2) added by the Hubbard interaction, which is back to the Anderson model if $t_{1 \sigma}=\tilde{t}_{1 \sigma} \equiv t_{1}$. In the meanwhile, $V_{l \sigma}$ term takes into account the finite range interactions between the impurity orbital and conduction electrons $\left(l=0, \ldots, N_{f}\right)$. Without losing the generality, we consider the screening channels as the spinless fermions and take $V_{l \sigma}=\tilde{V}_{l \sigma}=V_{l}$ for $l=1, . ., N_{f}$ for convenience. The $V_{x \sigma l}$ and $V_{y l}$ terms describe the spin exchange interactions. We take those with $l=1, \ldots, N_{f}$ vanishing for convenience. The $t_{2}$ term which is newly introduced in the Hamiltonian represents that two hybridizing channel electrons with opposite spins hop onto the impurity orbital simultaneously. To renormalize the model it is proper that this term as well as spin exchange terms are included in the Hamiltonian.

In (2.3), if the coupling constants with the 'tilde' are equal to those without the 'tilde', the Hamiltonian is reduced to the $\mathrm{PH}$ symmetric one under $\epsilon_{d}=-U / 2$. As we have shown that for this reduced model the consistency of the renormalization group equations forces the PH symmetry have to hold [23]. This Hamiltonian also includes the Kondo model as a special case [23]. Spinless models can also be reached by a proper choice of the coupling constants.

We shall show there is a FL-NFL phase transition for the system. To understand the physical mechanics of the appearance of the phase transition, we separate the Hamiltonian (2.3) into two parts, as done by Zhang, Yu and Su 17] for the spinless impurity model, $H=H_{h}+H_{s}$, where

$$
\begin{aligned}
H_{h} & =\sum_{k>0, \sigma} \epsilon_{k} c_{k \sigma}^{\dagger} c_{k \sigma}+\epsilon_{d} n_{d}+U n_{d \uparrow} n_{d \downarrow} \\
& +\frac{1}{\sqrt{L}} \sum_{\sigma}\left[\left(t_{1 \sigma}\left(1-n_{d}\right)+\tilde{t}_{1 \sigma} n_{d}\right) c_{\sigma}^{\dagger} d_{\sigma}+h . c .\right] \\
& +\frac{t_{2}}{L} \sum_{\sigma}\left(c_{\sigma}^{\dagger} c_{-\sigma}^{\dagger} d_{\sigma} d_{-\sigma}+h . c .\right) \\
& +\frac{1}{L} \sum_{\sigma}\left[V_{x \sigma}\left(2-n_{d}\right)+\tilde{V}_{x \sigma}\left(n_{d}-1\right)\right] c_{\sigma}^{\dagger} c_{\sigma} d_{\sigma}^{\dagger} d_{\sigma} \\
& +\frac{1}{L} \sum_{\sigma} V_{y} c_{\sigma}^{\dagger} c_{-\sigma} d_{-\sigma}^{\dagger} d_{\sigma} \\
& +\frac{1}{L} \sum_{\sigma, \sigma^{\prime}}\left[V_{0 \sigma}\left(2-n_{d}\right)+\tilde{V}_{0 \sigma}\left(n_{d}-1\right)\right] c_{\sigma}^{\dagger} c_{\sigma} d_{\sigma^{\prime}}^{\dagger} d_{\sigma^{\prime}}
\end{aligned}
$$

accordance with which, we can define standard bosonic creation and annihilation operators

$$
b_{k l}=-i \sqrt{\frac{1}{k}} \rho_{l}(k) \quad, \quad b_{k l}^{\dagger}=i \sqrt{\frac{1}{k}} \rho_{l}(-k) .
$$

The screening electron operator is represented, via the bosonic operator, as

$$
\begin{aligned}
& c_{l}^{\dagger}(x)=\frac{1}{\sqrt{\tau_{0}}} \exp \left[-i \phi_{l}(x)\right], \\
& \phi_{l}(x)=\sum \sqrt{\frac{1}{k L}}\left(b_{k l}^{\dagger} e^{-i k x}+b_{k l} e^{i k x}\right),
\end{aligned}
$$

and the screening Hamiltonian, $H_{s}$, is transformed to 


$$
H_{s}=\sum_{k, l>0} \frac{k}{\rho} b_{k l}^{\dagger} b_{k l}-\sum_{k, l>0} \frac{i V_{l}}{\sqrt{L}} \sqrt{k}\left(b_{k l}^{\dagger}-b_{k l}\right) n_{d},
$$

which can be diagonalized by the following canonical transformation

$$
T=\exp \left\{i \sum_{k, l>0} \frac{\rho V_{l}}{\sqrt{k L}}\left(b_{k l}^{\dagger}+b_{k l}\right) n_{d}\right\} .
$$

Since this canonical transformation does not commute with $d_{\sigma}$, the hybridization terms are transformed to the coupling forms among the impurity, hybridizing and screening channels while other terms do not change in $H_{h}$. We have the effective Hamiltonian, $H=H_{0}+H_{I}$, with

$$
\begin{aligned}
H_{0} & =\sum_{k>0, \sigma} \epsilon_{k} c_{k \sigma}^{\dagger} c_{k \sigma}+\epsilon_{d} n_{d}+U n_{d \uparrow} n_{d \downarrow} \\
& +\frac{1}{L} \sum_{\sigma, \sigma^{\prime}}\left[V_{0 \sigma}\left(2-n_{d}\right)+\tilde{V}_{0 \sigma}\left(n_{d}-1\right)\right] c_{\sigma}^{\dagger} c_{\sigma} d_{\sigma^{\prime}}^{\dagger} d_{\sigma^{\prime}} \\
& +\frac{1}{L} \sum_{\sigma}\left[V_{x \sigma}\left(2-n_{d}\right)+\tilde{V}_{x \sigma}\left(n_{d}-1\right)\right] c_{\sigma}^{\dagger} c_{\sigma} d_{\sigma}^{\dagger} d_{\sigma} \\
& +\sum_{k, l>0} \frac{k}{\rho} b_{k l}^{\dagger} b_{k l}, \\
H_{I} & =\frac{1}{\sqrt{L}} \sum_{\sigma}\left[\left(t_{1 \sigma}\left(1-n_{d}\right)+\tilde{t}_{1 \sigma} n_{d}\right) \Delta^{\dagger} c_{\sigma}^{\dagger} d_{\sigma}+h . c .\right] \\
& +\frac{t_{2}}{L} \sum_{\sigma}\left(\Delta^{\dagger 2} c_{\sigma}^{\dagger} c_{-\sigma}^{\dagger} d_{\sigma} d_{-\sigma}+h . c .\right) \\
& +\frac{V_{y}}{L} \sum_{\sigma} c_{\sigma}^{\dagger} c_{-\sigma} d_{-\sigma}^{\dagger} d_{\sigma} .
\end{aligned}
$$

The operator $\Delta^{\dagger}$ is given by $\Delta^{\dagger}=\exp \left\{\sum_{k, l>0} \frac{i \rho V_{l}}{\sqrt{k L}}\left(b_{k l}^{\dagger}+\right.\right.$ $\left.\left.b_{k l}\right)\right\}$ or in the coordinate representation $\Delta^{\dagger}(x)=$ $\exp \left\{i \sum \frac{\delta_{l}}{\pi} \phi_{l}(x)\right\}$ where $\delta_{l}=\pi \rho V_{l}$.

Now, we turn to derive the partition function of the Hamiltonian (3.3), where $H_{0}$ and $H_{I}$ are considered as the unperturbed Hamiltonian and the interaction Hamiltonian, respectively. Hence, we have a partition function

$$
Z=\operatorname{Tr}\left(e^{-\beta H}\right)=\operatorname{Tr}\left(e^{-\beta H_{0}} T_{\tau} \exp \left\{-\int_{0}^{\beta} H_{I}(\tau) d \tau\right\}\right),
$$

where $\beta$ is the inverse of the temperature, $T_{\tau}$ is the (imaginary) time ordering symbol and $H_{I}(\tau)=e^{H_{0} \tau} H_{I} e^{-H_{0} \tau}$ is the interaction-picture operator of $H_{I}$. Setting a sequence of times $0<\tau_{1}<\ldots<\tau_{n}<\beta$, the partition function is expanded as $Z=\sum_{n=o}^{\infty} Z_{n}$, where

$Z_{n}=(-1)^{n} \int_{0}^{\beta} d \tau_{n} \ldots \int_{0}^{\tau_{2}} d \tau_{1} \operatorname{Tr}\left(e^{-\beta H_{0}} H_{I}\left(\tau_{n}\right) \ldots H_{I}\left(\tau_{1}\right)\right)$
The Hilbert space of $H_{0}$ can be projected to four subspaces characterized by four impurity electron states, $|\alpha\rangle=|0\rangle, \mid \sigma>$ and $|3>\equiv| \uparrow \downarrow\rangle$. Each term in the interaction $H_{I}$ plays a role of the 'dipole' operator causing a transition between those sub-spaces. Such a kind of problems can be treated in Haldane's familiar way [30]: Write the partition function in terms of a sum over histories of the impurity. We will follow the formalism given by Si and Kotliar 18,25. Setting $P_{\alpha}=|\alpha><\alpha|$ is a projection operator to $\alpha$-state subspace, $H_{0}$, then, is projected as

$$
H_{0}=\sum_{\alpha} H_{\alpha} P_{\alpha}
$$

where

$$
\begin{aligned}
H_{\alpha} & =\epsilon_{d, \alpha}+U_{\alpha}+\sum_{\sigma} V_{\alpha}^{\sigma} c_{\sigma}^{\dagger} c_{\sigma} \\
& +\sum_{k>0 ; \sigma} \epsilon_{k} c_{k \sigma}^{\dagger} c_{k \sigma}+\sum_{k, l>0} \frac{k}{\rho} b_{k l}^{\dagger} b_{k l} .
\end{aligned}
$$

The impurity levels, here, are projected as $\epsilon_{d, 0}=0$, $\epsilon_{d, \sigma}=\epsilon_{d}$ and $\epsilon_{d, 3}=2 \epsilon_{d}$; the Hubbard interactions are $U_{0}=U_{\sigma}=0$ and $U_{3}=U$. Making a shift of the ground state energy, we can redefine $\epsilon_{d, \alpha}+U_{\alpha}$ as $\varepsilon_{\alpha}$ with $\varepsilon_{0}=-\epsilon_{d}-U / 4, \varepsilon_{\sigma}=-U / 4$ and $\varepsilon_{3}=\epsilon_{d}+3 U / 4$, which obey $\sum \varepsilon_{\alpha}=0$. The potentials are given by

$$
V_{0}^{\sigma}=0, \quad V_{\sigma^{\prime}}^{\sigma}=\frac{V_{0 \sigma}}{L}+\frac{V_{x \sigma}}{L} \delta_{\sigma \sigma^{\prime}}, \quad V_{3}^{\sigma}=\frac{2 \tilde{V}_{0 \sigma}}{L}+\frac{\tilde{V}_{x \sigma}}{L} .
$$

Now, we sum over the impurity states by inserting a complete set of the impurity states at every discrete time. Thus, (3.5) is rewritten as

$$
\begin{aligned}
Z_{n}= & (-1)^{n} \sum_{\alpha_{1}, \alpha_{2}, \ldots, \alpha_{n}} \int_{0}^{\beta} d \tau_{n} \ldots \int_{o}^{\tau_{2}} d \tau_{1} \\
\times & g_{\alpha_{n+1} \alpha_{n}}^{\prime} \ldots g_{\alpha_{2} \alpha_{1}}^{\prime} \\
\times & t r_{c}\left\{e^{-H_{\alpha_{n+1}}\left(\beta-\tau_{n}\right)} O^{\prime}\left(\alpha_{n+1}, \alpha_{n}\right) e^{-H_{\alpha_{n}}\left(\tau_{n}-\tau_{n-1}\right)}\right. \\
& \left.\ldots e^{-H_{\alpha_{2}}\left(\tau_{2}-\tau_{1}\right)} O^{\prime}\left(\alpha_{2}, \alpha_{1}\right) e^{-H_{\alpha_{1}}}\right\}
\end{aligned}
$$

where $t r_{c}$ indicates the trace carried out for the conduction band. $\alpha_{1}, \ldots, \alpha_{n}$ and $\tau_{1}, \ldots, \tau_{n}$ label a Feynman trajectory with $\alpha_{n+1}=\alpha_{1} . g_{\alpha \beta}^{\prime}$ are coupling constants, $g_{0 \sigma}^{\prime}\left(g_{-\sigma 3}^{\prime}\right) \equiv g_{1 \sigma}^{\prime}\left(\tilde{g}_{1 \sigma}^{\prime}\right)=t_{1 \sigma} / \sqrt{L}\left(\tilde{t}_{1 \sigma} / \sqrt{L}\right), g_{\sigma,-\sigma}^{\prime} \equiv g_{y}^{\prime}=$ $V_{y} / L$ and $g_{03}^{\prime} \equiv g_{2}^{\prime}=t_{2} / L$. The 'dipole' operators $O^{\prime}(\alpha, \beta)$ are given by

$$
\begin{aligned}
& O^{\prime}(0, \sigma)=O^{\prime \dagger}(\sigma, 0) \\
& =O^{\prime}(-\sigma, 3)=O^{\prime \dagger}(3,-\sigma)=\Delta^{\dagger} c_{\sigma}^{\dagger}, \\
& O^{\prime}(0,3)=O^{\prime \dagger}(3,0)=\Delta^{\dagger 2} c_{\uparrow}^{\dagger} c_{\downarrow}^{\dagger}, \\
& O^{\prime}(\sigma,-\sigma)=c_{-\sigma}^{\dagger} c_{\sigma} .
\end{aligned}
$$


A similar bosonization procedure to the screening channels can be applied to the impurity channel. As a result, the projected Hamiltonian transforms into

$$
\begin{array}{r}
H_{\alpha}=H_{c}+\varepsilon_{\alpha}-\sum_{k>0 ; \sigma} \frac{i \delta_{\alpha}^{\sigma}}{\pi \rho} \sqrt{\frac{k}{L}}\left(b_{k \sigma}^{\dagger}-b_{k \sigma}\right), \\
H_{c}=\sum_{k>0 ; \rho} \frac{k}{\rho} b_{k \sigma}^{\dagger} b_{k \sigma}+\sum_{k, l>0} \frac{k}{\rho} b_{k l}^{\dagger} b_{k l},
\end{array}
$$

where $b_{k \sigma}$, the bosonic operator corresponding to $c_{k \sigma}$, is given through

$$
\begin{aligned}
& c_{\sigma}^{\dagger}(x)=\exp \left[-i \phi_{\sigma}(x)\right], \\
& \phi_{\sigma}(x)=\sum_{k} \sqrt{\frac{1}{k L}}\left(b_{k \sigma}^{\dagger} e^{-i k x}+b_{k \sigma} e^{i k x}\right) .
\end{aligned}
$$

The phase shifts $\delta_{\alpha}^{\sigma}=\pi \rho V_{\alpha}^{\sigma} L$ are defined by

$$
\delta_{0}^{\sigma}=0, \quad \delta_{\sigma^{\prime}}^{\sigma}=\delta_{0}+\delta_{x \sigma} \delta_{\sigma^{\prime} \sigma}, \quad \delta_{3}^{\sigma}=2 \tilde{\delta}_{0}+\tilde{\delta}_{x \sigma},
$$

where $\delta_{0}=\pi \rho V_{0 \sigma}, \tilde{\delta}_{0}=\pi \rho \tilde{V}_{0 \sigma}, \delta_{x \sigma}=\pi \rho V_{x \sigma}$ and $\tilde{\delta}_{x \sigma}=\pi \rho \tilde{V}_{x \sigma}$ are the Born approximation values of the general phase shifts $2 \tan ^{-1}\left(\pi \rho V_{0 \sigma} / 2\right)$, and so on. The Hamiltonian (3.10) can also be diagonalized to $H_{c}+\varepsilon_{\alpha}$ by the following canonical transformations

$$
U_{\alpha}=\Pi_{\sigma} U_{\delta_{\alpha}^{\sigma}}=\Pi_{\sigma} \exp \left\{i \delta_{\alpha}^{\sigma} \phi_{\sigma}\right\} .
$$

Meanwhile, the bosonized 'dipole' operators can be transformed to

$$
O(\alpha, \beta)=\exp \left\{i q_{\alpha \beta}^{a} \phi_{a}\right\},
$$

where $\phi_{a}=\left(\phi_{\sigma}, \phi_{l}\right)$. The quantities $q_{\alpha \beta}^{a}$, which will be called 'charges', are

$$
\begin{aligned}
q_{0 \sigma}^{a} & =\left(\left(\frac{\delta_{x \sigma^{\prime}}}{\pi}-1\right) \delta_{\sigma \sigma^{\prime}}+\frac{\delta_{0}}{\pi}, \frac{\delta_{l}}{\pi}\right), \\
q_{\sigma-\sigma}^{a} & =\left(\left(\frac{\delta_{x \sigma^{\prime}}}{\pi}-1\right)\left(\delta_{\sigma^{\prime}-\sigma}-\delta_{\sigma^{\prime} \sigma}\right), 0\right), \\
q_{-\sigma 3}^{a} & =\left(\left(\frac{\delta_{x \sigma^{\prime}}}{\pi}-1\right) \delta_{\sigma^{\prime}, \sigma}+\frac{\delta_{0}}{\pi}\right. \\
& \left.+\frac{1}{\pi}\left(\tilde{\delta}_{x \sigma^{\prime}}-\delta_{x \sigma^{\prime}}+2 \tilde{\delta}_{0}-2 \delta_{0}\right), \frac{\delta_{l}}{\pi}\right), \\
q_{03}^{a} & =\left(\frac{\tilde{\delta}_{x \sigma^{\prime}}+2 \tilde{\delta}_{0}}{\pi}-1, \frac{2 \delta_{l}}{\pi}\right),
\end{aligned}
$$

where $a=\left(\sigma^{\prime}, l\right)$ and the 'charges' are anti-symmetric: $q_{\alpha \beta}^{a}=-q_{\beta \alpha}^{a}$. Using the bosonic 'dipole' operators (3.14) and defining the interaction-picture operators $O(\alpha, \beta)(\tau) \equiv e^{H_{c} \tau} O(\alpha, \beta) e^{-H_{c} \tau}$, the partition function reads

$$
\begin{aligned}
Z_{n} & =(-1)^{n} \sum_{\alpha_{1}, \ldots, \alpha_{n}} \int_{0}^{\beta-\tau_{0}} \frac{d \tau_{n}}{\tau_{0}} \ldots \int_{0}^{\tau_{2}-\tau_{0}} \frac{d \tau_{1}}{\tau_{0}} \\
& \times g_{\alpha_{n+1} \alpha_{n} \ldots g_{\alpha_{2} \alpha_{1}}} \exp \left\{-\sum_{i} E_{\alpha_{i}+1} \frac{\tau_{i+1}-\tau_{i}}{\tau_{0}}\right\} \\
& \times \operatorname{tr}_{c}\left(e^{-H_{c} \beta} O\left(\alpha_{n+1}, \alpha_{n}\right)\left(\tau_{n}\right) \ldots O\left(\alpha_{2}, \alpha_{1}\right)\left(\tau_{1}\right)\right),
\end{aligned}
$$

where $g_{\alpha \beta}=g_{\alpha \beta}^{\prime} \tau_{0}$ are dimensionless coupling constants and $E_{\alpha}=\varepsilon_{\alpha} \tau_{0}$ are regarded as dimensionless external fields. The trace part of (3.16) is just the correlation function of $n$-vertex operators. In the zero temperature limit, it can be calculated by using the free boson field theory. The result is given rise to:

$$
\begin{aligned}
Z & =\sum_{n} \sum_{\alpha_{1}, \ldots, \alpha_{n}} \int_{0}^{\beta-\tau_{0}} \frac{d \tau_{n}}{\tau_{0}} \ldots \int_{0}^{\tau_{2}-\tau_{0}} \frac{d \tau_{1}}{\tau_{0}} \\
& \times \exp \left(-S\left[\tau_{1}, \ldots, \tau_{n} ; \alpha_{1}, \ldots, \alpha_{n}\right]\right), \\
S[\tau, \alpha] & =\sum_{i<j} \sum_{a=\sigma, l} q_{\alpha_{i} \alpha_{i+1}}^{a} q_{\alpha_{j} \alpha_{j+1}}^{a} \ln \frac{\tau_{j}-\tau_{i}}{\tau_{0}} \\
& -\sum_{i} \ln \left(g_{\alpha_{i+1} \alpha_{i}}\right)+\sum_{i} E_{\alpha_{i+1}} \frac{\tau_{i+1}-\tau_{i}}{\tau_{0}},
\end{aligned}
$$

which may be regarded to describe a one dimensional multi-component plasma of kinks with 'charges' $q_{\alpha \beta}^{a}$ and 'fugacities' $g_{\alpha \beta} . E_{\alpha}$ is an 'external field'.

\section{B. Related to Charge-Spin Chain and Transitivity of 'Dipole' operators}

The partition function (3.17) describes a multicomponent kink plasma with logarithmic interactions. There are six types of kinks which correspond to the different pairs of the impurity states, the charge pairs $(0 \sigma),(\sigma 3)$ and $(03)$ and the spin pair $(\uparrow \downarrow)$. This leads to the logarithmic interactions between the kink-pairs can be transformed to the interactions among the charge- and spin-pairs. To reach this point we note that the 'charges' of kinks are of their transitivity:

$$
q_{\alpha \beta}^{a}+q_{\beta \gamma}^{a}=q_{\alpha \gamma}^{a}
$$

This leads to the rewriting of the action $S[\tau, \alpha]$, i. e.

$$
\begin{aligned}
S[\tau, \alpha] & =\sum_{i<j} \tilde{K}\left(\alpha_{i}, \alpha_{i+1} ; \alpha_{j}, \alpha_{j+1}\right) \ln \frac{\tau_{j}-\tau_{i}}{\tau_{0}} \\
& -\sum_{i} \ln \left(g_{\alpha_{i+1} \alpha_{i}}\right)+\sum_{i} E_{\alpha_{i+1}} \frac{\tau_{i+1}-\tau_{i}}{\tau_{0}}
\end{aligned}
$$

where

$$
\begin{aligned}
& \tilde{K}\left(\alpha_{i}, \alpha_{i+1} ; \alpha_{j}, \alpha_{j+1}\right)=K\left(\alpha_{i}, \alpha_{j}\right) \\
& +K\left(\alpha_{i+1}, \alpha_{j+1}\right)-K\left(\alpha_{i}, \alpha_{j+1}\right)-K\left(\alpha_{i+1}, \alpha_{j}\right),
\end{aligned}
$$

where $K(\alpha, \beta)$, called charge- and spin- stiffness constants, are defined by [31]

$$
K(\alpha, \beta)=-\frac{1}{2} \sum_{a}\left(q_{\alpha \beta}^{a}\right)^{2} .
$$


The model described by (3.20) is equivalent to the onedimensional charge-spin chain model with $1 / \tau^{2}$ interaction 24] and incorporating the effective fields 25]:

$$
\begin{aligned}
S[\alpha, \tau] & =\sum_{i<j} K\left(\alpha_{i}, \alpha_{j}\right) \frac{\tau_{0}^{2}}{\left(\tau_{j}-\tau_{i}\right)^{2}}-\sum_{i} \ln \left(g_{\alpha_{i+1} \alpha_{i}}\right) \\
& +\sum_{i} E_{\alpha_{i+1}} \frac{\tau_{i+1}-\tau_{i}}{\tau_{0}}
\end{aligned}
$$

The model is just a special case of the general onedimensional model with $1 / r^{2}$ interaction considered by Cardy 24. It is worth to point out that the transitivity (3.19) plays an important role to reach Cardy's model. In fact, the transitivity of the 'charges' stems from the transitivity of the 'dipole' operators:

$$
O(\alpha, \beta) O(\beta, \gamma)=O(\alpha, \gamma)
$$

This transitivity implies that all 'dipole' operators together with the vanishing operator $\phi$ and the identity operator $I$ form a semigroup under the operation: Either $O(\alpha, \beta) O(\gamma, \delta)=\phi$ for $\beta \neq \gamma$ or the transitivity (3.24). Physically, the semigroup relates to the transitivity of the charge-spin-flips: e.g., the pair (03) can be separated as two pairs $(0 \sigma)$ and $(\sigma 3)$, etc. If the transitivity of the charge-spin-flips is broken, the charge-spin chain is interrupted. Therefore, the model is not selfconsistent. Originally, the transitivity comes from the two-body hybridization and the spin-exchange interaction in the Hamiltonian (2.3). Therefore, the inclusion of them in 2.3) is proper to the self-consistency of the model. In the next section, we shall see that the correct renormalization flows mustn't exclude those of the fugacities $g_{\uparrow \downarrow}$ and $g_{03}$.

To conclude this section, we would like to mention that the transitivity of the 'charges' and their anti-symmetry leads to Jacobi identities, which implies the existence of a Lie algebra. We arrange an appendix ( appendix A) to discuss the properties of the Lie algebra.

\section{PHASE STRUCTURES}

In this section, we study the phase structures of the models by using the AYHC poor man's scaling theory. The details of the derivation of the renormalization group equations have been given in Appendix B. Using those equations, we analyze the phase structures of the general model and various reduced models.

\section{A. General Model}

In the most general case, six non-zero fugacities $g_{\alpha \beta}$ are independent of each other. Furthermore, six stiffness constants are the functions of six phase shifts $\delta_{0}, \tilde{\delta}_{0}, \delta_{x \sigma}$ and $\tilde{\delta}_{x \sigma}$ in the quadrate. The six stiffness constants are also independent of each other. To see this point, we consider the small phase shifts. In this case (3.22) expresses a non-singular linear transformation if the quadrate are neglected. Thus, the renormalization group equations are exactly the 4-state example of the Cardy's general results 24 and their symmetry breaking generalization 25] (also see Appendix B):

$$
\begin{aligned}
\frac{d g_{1 \sigma}}{d l n \tau_{0}} & =\left(1-K_{0 \sigma}\right) g_{1 \sigma}+g_{1,-\sigma} g_{y} e^{-E_{-\sigma}+\frac{1}{2}\left(E_{0}+E_{\sigma}\right)} \\
& +g_{2} \tilde{g}_{1-\sigma} e^{-E_{3}+\frac{1}{2}\left(E_{0}+E_{\sigma}\right)} \\
\frac{d g_{y}}{d l n \tau_{0}} & =\left(1-K_{\sigma,-\sigma}\right) g_{y}+g_{1 \sigma} g_{1,-\sigma} e^{-E_{0}+\frac{1}{2}\left(E_{0}+E_{-\sigma}\right)} \\
& +\tilde{g}_{1 \sigma} \tilde{g}_{1,-\sigma} e^{-E_{3}+\frac{1}{2}\left(E_{0}+E_{-\sigma}\right)} \\
\frac{d \tilde{g}_{1 \sigma}}{d l n \tau_{0}} & =\left(1-K_{-\sigma 3}\right) \tilde{g}_{1 \sigma}+g_{1-\sigma} g_{2} e^{-E_{0}+\frac{1}{2}\left(E_{3}+E_{-\sigma}\right)} \\
& +g_{y} \tilde{g}_{1,-\sigma} e^{-E_{\sigma}+\frac{1}{2}\left(E_{3}+E_{-\sigma}\right)} \\
\frac{d g_{2}}{d l n \tau_{0}} & =\left(1-K_{03}\right) g_{2}+\sum_{\sigma} g_{1 \sigma} \tilde{g}_{1-\sigma} e^{-E_{\sigma}+\frac{1}{2}\left(E_{0}+E_{3}\right)}
\end{aligned}
$$

for the fugacities. Here $K_{\alpha \beta}=-K(\alpha, \beta)$. For the external fields, i. e. $U$ and $\epsilon_{d}$, one has

$$
\begin{aligned}
\tau_{0} \frac{d \epsilon_{d}}{d l n \tau_{0}} & =\tau_{0} \epsilon_{d}+\left(g_{1 \uparrow}^{2}+g_{1 \downarrow}^{2}\right) e^{E_{0}-E_{\uparrow}} \\
& -\frac{1}{2}\left(g_{1 \uparrow}^{2}+g_{1 \downarrow}^{2}\right) e^{E_{\uparrow}-E_{0}} \\
& -\frac{1}{2}\left(\tilde{g}_{1 \uparrow}^{2}+\tilde{g}_{1 \downarrow}^{2}\right) e^{E_{\uparrow}-E_{3}}+g_{2}^{2} e^{E_{0}-E_{3}}-g_{y}^{2}, \\
\tau_{0} \frac{d U}{d l n \tau_{0}} & =\tau_{0} U+\left(g_{1 \uparrow}^{2}+g_{1 \downarrow}^{2}\right)\left(e^{E_{\uparrow}-E_{0}}-e^{E_{0}-E_{\uparrow}}\right)+2 g_{y}^{2} \\
& +\left(\tilde{g}_{1 \uparrow}^{2}+\tilde{g}_{1 \downarrow}^{2}\right)\left(e^{E_{\uparrow}-E_{3}}-e^{E_{3}-E_{\uparrow}}\right) \\
& -g_{2}^{2}\left(e^{E_{0}-E_{3}}+e^{E_{3}-E_{0}}\right) .
\end{aligned}
$$

For the stiffness constants, one has

$$
\begin{aligned}
\frac{d K_{\alpha \beta}}{d l n \tau_{0}} & =-\sum_{\gamma} g_{\alpha \gamma}^{2} e^{E_{\alpha}-E_{\gamma}}\left(K_{\alpha \beta}+K_{\alpha \gamma}-K_{\beta \gamma}\right) \\
& -\sum_{\gamma} g_{\beta \gamma}^{2} e^{E_{\beta}-E_{\gamma}}\left(K_{\alpha \beta}+K_{\beta \gamma}-K_{\alpha \gamma}\right) .
\end{aligned}
$$

We make a few comments on the renormalization group equations (4.1), (4.2) and (4.3).

(i) As we have seen, if the model can be mapped to the special model of Cardy's, $V_{y}$ and $t_{2}$ can not vanish. Furthermore, from (4.1), we see that even we start from the vanishing initial values of $g_{2}$ and $g_{y}$, they will increase as $g_{1 \sigma}$ and $\tilde{g}_{1 \sigma}$. This means that the flows of the vanishing values of them are not correct renormalization flows.

(ii) Since our renormalization group is perturbative in its treatment of $g_{\alpha \beta}$, the phase structure of the system can be described by $g_{\alpha \beta}$. The strong-coupling fixed points in the quantum impurity problem are outside the range of validity of the AYHC poor man's scaling theory, but it can give the correct flow directions. In the strongly 
coupling limit, the fixed point behaviors need to be more carefully discussed.

(iii) Near the fixed point, $\epsilon_{d}, U \rightarrow 0$ in the order of $g_{\alpha \beta}^{2}$. Therefore, we can regard all $e^{E_{\alpha}}=1$ when we discuss the behaviors near the fixed point. Then, we can rewrite the renormalization group equations in the form of perturbative $g_{\alpha \beta}$ as follows:

$$
\begin{aligned}
& \frac{d g_{1 \sigma}}{d l n \tau_{0}}=\left(1-K_{0 \sigma}\right) g_{1 \sigma}+g_{1,-\sigma} g_{y}+g_{2} \tilde{g}_{1-\sigma}, \\
& \frac{d g_{y}}{d l n \tau_{0}}=\left(1-K_{\sigma,-\sigma}\right) g_{y}+g_{1 \sigma} g_{1,-\sigma}+\tilde{g}_{1 \sigma} \tilde{g}_{1,-\sigma}, \\
& \frac{d \tilde{g}_{1 \sigma}}{d l n \tau_{0}}=\left(1-K_{-\sigma 3}\right) \tilde{g}_{1 \sigma}+g_{1-\sigma} g_{2}+g_{y} \tilde{g}_{1,-\sigma}, \\
& \frac{d g_{2}}{d l n \tau_{0}}=\left(1-K_{03}\right) g_{2}+\sum_{\sigma} g_{1 \sigma} \tilde{g}_{1-\sigma}, \\
& \tau_{0} \frac{d \epsilon_{d}}{d l n \tau_{0}}=\tau_{0} \epsilon_{d}+\frac{1}{2}\left(g_{1 \uparrow}^{2}+g_{1 \downarrow}^{2}-\tilde{g}_{1 \uparrow}^{2}\right. \\
&\left.\quad-\tilde{g}_{1 \downarrow}^{2}\right)+g_{2}^{2}-g_{y}^{2}, \\
& \tau_{0} \frac{d U}{d l n \tau_{0}}=\tau_{0} U+2\left(g_{y}^{2}-g_{2}^{2}\right) . \\
& \frac{d K_{\alpha \beta}}{d l n \tau_{0}}=-\sum_{\gamma} g_{\alpha \gamma}^{2}\left(K_{\alpha \beta}+K_{\alpha \gamma}\right. \\
&\left.\quad-K_{\beta \gamma}\right)-\sum_{\gamma} g_{\beta \gamma}^{2}\left(K_{\alpha \beta}+K_{\beta \gamma}-K_{\alpha \gamma}\right) .
\end{aligned}
$$

Under those considerations and according to 4.4, we can describe the phase structure of the system.

(i) For all $K_{\alpha \beta}>1$, all fugacities are irrelevant and renormalized to zero. There exists a weak coupling fixed point $g_{\alpha \beta}^{*}=0$. The fixed point Hamiltonian is similar to that of the multi-channel X-ray edge problem [22. The system exhibits a power law decay of the correlation function with a non-universal exponent. This is a NFL phase. By the definitions of $K_{0 \sigma}$, the existence of the screening channels ensures the possibility of $K_{0 \sigma}>1$ and then reaches the NFL phase. The one-channel model in a small exchange interaction can not have this phase [17].

(ii) Once one of the coupling constant becomes relevant, the low temperature fixed point of the system, in general, is Fermi-liquid-like. It is still possible that there is a plenty of phase structures at finite temperature. Because all $K_{\alpha \beta}$ are independent of each other, in general there will be a unique smallest $K_{\alpha \beta}$ with $(\alpha \beta)=\left(\alpha_{0} \beta_{0}\right)$. When $K_{\alpha_{0} \beta_{0}}$ decreases to 1 and then smaller than 1, a phase transition happens. There are such six phases that $g_{\alpha_{0} \beta_{0}}$ is relevant and all the $g_{\alpha \beta} \neq g_{\alpha_{0} \beta_{0}}$ are irrelevant in each phase. They may have different finite temperature behaviors. For example, we consider $g_{y}$-relevant phase. We focus on the fixed point Hamiltonian. Introducing the scaled parameters $V_{y} / L=J_{\perp} / 2, V_{0 \sigma} / L=-J_{z} / 4$ and $V_{x \sigma} / L=J_{z} / 2$ with $J_{z}>0$ (this ensures $K_{\uparrow \downarrow}<1$ ), we see the fixed point Hamiltonian $H^{*}$ is just the onechannel Kondo Hamiltonian:

$$
\begin{aligned}
H^{*} & =\sum_{k>0, \sigma} \epsilon_{k} c_{k \sigma}^{\dagger} c_{k \sigma}+\epsilon_{d} n_{d}+U n_{d \uparrow} n_{d \downarrow} \\
& +\sum_{k>o, l=1} \frac{k}{\rho} b_{k l}^{\dagger} b_{k l}+\frac{J_{\perp}}{2}\left(S_{c}^{+} s_{d}^{-}+h . c .\right)+J_{z} S_{c}^{z} s_{d}^{z} .
\end{aligned}
$$

Here $\mathbf{S}_{c}$ and $\mathbf{s}_{d}$ are the spin operators of the hybridizing and local electrons respectively. The $g_{y}\left(J_{\perp}\right)$-relevant phase is controlled by the strong fixed point of Kondo problem. Furthermore, the values of $V_{0 \sigma}$ and $V_{x \sigma}$ are not important as long as they are within the parameter regime of the $g_{y}$-relevant phase. Hence, the fixed point corresponding to $g_{y}$-relevant phase is the FL, which is a collective mode of the system and transfers to a state with a local moment at $T>T_{K}$, the Kondo temperature. Equations (4.5) is reduced to

$$
\tau_{0} \frac{d \epsilon_{d}}{d \ln \tau_{0}}=\tau_{0} \epsilon_{d}-g_{y}^{2}, \quad \tau_{0} \frac{d U}{d \ln \tau_{0}}=\tau_{0} U+2 g_{y}^{2},
$$

which imply that $U$ increase positively if its initial value is not negative. This repeats the results of Anderson model: When the impurity level width $\Gamma \sim t_{1}^{2}$ is much less than $U$, the model is equivalent to the Kondo model [12].

For the $g_{1 \sigma}\left(\tilde{g}_{1 \sigma}\right)$-relevant phase, according to (4.5), $U$ keeps to be almost invariant if its initial value is the same as at the fixed point. Hence, $\Gamma \gg U$ in this case and the groundstate of the system may be read off from the exactly solvable Fano-Anderson model. It is a FL but there is no a local moment in excitation states. So we call this phase the free-orbital phase [13].

For the $g_{2}$-relevant phase, the empty and doublyoccupied states favor over singly-occupied states. The fixed point Hamiltonian $H^{*}=H_{0}^{*}+H_{I}^{*}$ where $H_{0}^{*}$ can be read off from (3.3) with replacing the all of parameters by their fixed point values and $H_{I}^{*}$ has a leading term $R \sim\left(g_{2}^{*} / \tau_{0}\right) \sum_{\sigma}\left(c_{\sigma}^{\dagger} c_{-\sigma}^{\dagger} d_{\sigma} d_{-\sigma}+\right.$ h.c. $)$. The matrix elements of $R$ in the fixed point can be evaluated by a similar considerations in the X-ray edge problem [33]. As done in 115], define $\mid n_{d}=0>$ to be the eigenstates of $\left.H_{0}^{*}\right|_{n_{d}=0}$ and $\mid n_{d}=2>$ to be $\left.H^{*}\right|_{n_{d}=2}$ 's. Then

$$
<n_{d}=0|R| n_{d}=2>\sim \tau^{\alpha} .
$$

The anomalous exponent $\alpha=\left(1-K_{03}\right) / 2$. (If $\alpha<0$ the operator $R$ is irrelevant, and the fixed point is the NFL as we have discussed.) In the present parameter regime, $\alpha>0$ and the operator $R$ are relevant. The fixed point is regarded as the FL 15$]$.

Thus, all phases with one relevant fugacity are FL but they may have different finite temperature behaviors. A FL-NFL phase transition occurs as the model parameters vary.

(iii) When several of the $K_{\alpha \beta} \leq 1$, the phase transition can be characterized by Cardy's graphical method 
[24. Draw a graph whose vertices are labeled by the states $0, \sigma$ and 3 (see Figure 1(a)). A particular transition corresponds to drawing a set of the edges $(\alpha \beta)$ which express that those $K_{\alpha \beta} \leq 1$. When $K_{\alpha \beta}$ and $K_{\beta^{\prime} \gamma}$ become smaller than one, $g_{\alpha \beta}$ and $g_{\beta^{\prime} \gamma}$ are relevant. If $\beta \neq \beta^{\prime}, g_{\alpha \gamma}$ is still irrelevant as long as $K_{\alpha \gamma}>1$. Before the next phase transition happens, we change the edge linking the vertices $\alpha$ and $\beta$ to a new vertex and the edge of $\beta^{\prime}$ and $\gamma$ to another vertex. Then repeat the previous process when other $K_{\alpha^{\prime} \gamma^{\prime}} \leq 1$. If $\beta=\beta^{\prime}$, even $K_{\alpha \gamma}>1$ the second and third terms of (4.1) also drive $g_{\alpha \gamma}$ to be relevant. Reflecting this point graphically, the vertices $\alpha, \beta$ and $\gamma$ are linked as an edge which is changed to a new vertex before the next phase transition appears. Cardy had explained the physical reason to cause $g_{\alpha \beta}$ becomes relevant [24]: Logarithmic interactions between kinks are no longer existent in the linked piece of the graph. Repeat the process till all the vertices are linked so that no further transitions are possible. Cardy calls the final phase high temperature (HT) phase, in which all the fugacities are relevant. Notice that the renormalization group equations are not valid quantitatively in the strongly coupling region. Therefore, the phase structure of the model in the relevant phase must be discussed more carefully. In some situations, the real phase diagram of the relevant phases is different from that given by the graphical method. To see this we now turn to various reduced models from the general model.

\section{B. Spin Symmetric Model}

The spin symmetric model is a model with $g_{\alpha \uparrow}=g_{\alpha \downarrow}$ and $K_{\alpha \uparrow}=K_{\alpha \downarrow}$. The phase structure of the system can be described graphically in Figure 1. Every diagram row of Figure 1 represents a sequence of transitions from the NFL phase to HT phase. The diagram consisting only of the vertices represents a phase while the diagram with lines is a critical point. For example, Figure 1(a) represents the sequence of transitions corresponding to $K_{0 \sigma}<K_{03}, K_{3 \sigma}, K_{\uparrow \downarrow}$. In fact, Fig. 1(a) and 1(b), 1(c) and $1(\mathrm{~d})$ reflect similar physical processes, respectively. The four-vertex phase in the figure is the NFL phase. The two-vertex phase of $1(\mathrm{a})(1(\mathrm{~b}))$ is the $g_{1 \sigma}\left(\tilde{g}_{1, \sigma}\right)$ and $g_{y}$ relevant phase. We arrive at the strong-coupling fixed point of the asymmetric Anderson model. This is the FL. The three-vertex phase of $1(\mathrm{c})(1(\mathrm{~d}))$ is controlled by the strong-coupling fixed point of the Kondo problem. And two-vertex phase is the same phase as 1(a)(1(b)). The three-vertex phase of $1(\mathrm{e})$ is the $g_{2}$-relevant phase, which is the FL. The phase structure of the spin symmetric model is different from that of the general model: The free orbital phase does not exist. Physically, since $g_{\alpha \uparrow}=g_{\alpha \downarrow}$ the spin can not be frozen out. Furthermore, when the $(0 \uparrow)$ kinks are bounded, the $(0 \downarrow)$ kinks are also bounded because of the spin symmetry, which leads to the $(\uparrow \downarrow)$ kinks are bounded too. Therefore, $g_{y}$ be- comes relevant together with $g_{1 \sigma}$ or $\tilde{g}_{1 \sigma}$. The free orbital condition, $\Gamma \gg U$, can not be satisfied in the spin symmetric model. We would like to mention a point that the spin symmetry does not change the dimensions of the Lie algebra described in Appendix A. Correspondingly, the largest number of the critical points in the sequences of the phase transition keeps to be invariant. As we have mentioned, in the strong coupling limit, the graphical method is not always valid quantitatively. Here this point reflected in the phase diagram is that the phase transitions from three-vertex phases to two-vertex phases do not really happen because both of the asymmetric Anderson model and the Kondo model have the same strongly coupling fixed point.

\section{Particle-Hole Symmetric Models}

Setting $g_{1 \sigma}=\tilde{g}_{1, \sigma}$ and $K_{0 \sigma}=K_{-\sigma 3}$ in the general model, the consistency of the renormalization flows of $g_{1 \sigma}$ and $\tilde{g}_{1 \sigma}$ forces that $E_{3}=E_{0}$ (as long as their initial values are equal). Thus, if the PH symmetry of the common hybridization term in the model is not broken, the consistency of the renormalization group equations requires that the system keeps the symmetry. The phase structure of the PH symmetric model is described in Figure 2. Since there is no spin symmetry, the free orbital phase exists (the two-vertex phase in Figs. 2(a) and 2(b)). The phase described by two-vertex diagrams in Figure 1 disappears because the model is PH symmetric. The Kondo phase and $g_{2}$-relevant phase still exist.

If we consider spin and PH symmetric model, the phase structure is shown by Figures 3(a),(b) and (c). This model has been discussed in [23]. Figures 3(a), (b) and (c) can be reexpressed more explicitly by Figure 3(d). Notice that there is no three critical point sequence of the transition in the $\mathrm{PH}$ symmetric model because it is impossible that $K_{\uparrow \downarrow}<K_{03}<K_{0 \sigma}$ and $K_{03}<K_{\uparrow \downarrow}<K_{0 \sigma}$. Physically, those sequences of transition break the $\mathrm{PH}$ symmetry and then are forbidden. Relating to the Lie algebra of the plasma 'charges', under the PH symmetry, the dimensions of the Lie algebra reduce to 3 . As we have done [23], the strongly coupling limit needs to be treated more carefully. The real phase diagram is shown in Figure 3(e). The phase of all fugacity relevance is divided into three parts: $I$ is still in the Kondo strongly coupling phase; $I I$ is the same as $G_{2}$-phase in which the empty and doubly occupied states are favored; and $I I I$ is all impurity state mixed phase.

\section{MIXED VALENCE STATE REGIME AND FIXED POINTS}

In the previous section, we term the FL and NFL states only in the sense that whether the infrared orthogonality catastrophe of the X-ray edge type appears. 
In fact, the situation may be subtler if the we consider the local state properties in the problem 18,25. To see this, we study the fixed points in the mixed valence state regimes, in which the renormalized impurity level shift $\epsilon_{d}^{*}$ is of the same order as the renormalized resonant width $\Gamma^{*}=\pi g_{t}^{* 2} / \tau$, i. e., $\left|\epsilon_{d}^{*}\right| \leq \Gamma^{*}$. Most interesting physical phenomena appear in the particle-hole symmetric model. At finite $U$, the particle-hole symmetry implies $\epsilon_{d}=-U / 2$ for their bare values. The renormalization group equations show that this constraint is kept after the renormalization except in the case of infinite $U$. So, the crossover between various regimes is still determined by the ratio $\left|\epsilon_{d}^{*}\right| / \Gamma^{*}$. We can still define the mixed valence states if $\left|\epsilon_{d}^{*}\right| \sim \Gamma^{*}$. On the other hand, the particle-hole symmetry reduces the number of the independent stiffness constants to two, $\gamma_{0}$ and $\gamma_{x}$. The fixed points in the $\gamma_{0}-\gamma_{x}$ plane can be figured out according to the stiffness constants $\epsilon_{t_{1}}=\frac{1}{2} \gamma_{0}, \epsilon_{x}=\gamma_{x} \epsilon_{t_{2}}=2 \gamma_{0}-\gamma_{x}$. The weak coupling mixed valence states is within the renormalized parameter regime $\epsilon_{t_{1}}^{*}>1, \epsilon_{x}^{*}>1$ and $\epsilon_{t_{2}}^{*}>1$. The strong coupling mixed valence states are corresponding to $\epsilon_{t_{1}}^{*}<1$ and $\epsilon_{t_{2}}^{*}<1$. There are two intermediate coupling mixed valence state regimes in which the renormalized stiffness constants are given by 1) $\epsilon_{t_{1}}^{*}<1, \epsilon_{x}^{*}<1$ and $\epsilon_{t_{2}}^{*}>1$ and 2) $\epsilon_{t_{1}}^{*}>1, \epsilon_{x}^{*}<1$ and $\epsilon_{t_{2}}^{*}<1$. The latter associates to the intermediate coupling mixed valence state we have already known in the infinite $U$ case 30,25 while the former to the $G_{2}$-irrelevant mixed valence states we newly find in the finite $U$ case here. We will not repeat the fixed point behaviors in the strong coupling case and the known intermediate coupling case but refer to the interested readers to read the relevant literatures 30,25]. What we would like to do is to review the weak coupling states and focus on the $G_{2}$-irrelevant states.

Within the weak coupling mixed valence regime, all running fugacities could be obtained by solving the linear renormalization group equations. As we have seen in the last section, there is a NFL fixed point in the sense of the X-ray edge infrared orthogonality catastrophe. Furthermore, in the mixed valence regime, there are also the violation of the Fermi liquid behavior in the calculation of the local single-particle and two-particle correlation functions. Some of them have been shown in refs. [18, 25] such as $G_{d c}, G_{d d}$ and two-particle excitonic and $d$-electron correlation functions as well as $d c$ correlation functions. Divergences appear in the $G_{d c}, G_{d d}$ and the $d c$ correlation functions, which shows the local NFL behavior. At the finite $U$, one may have more divergent correlation functions. For instance, the single $d$-electron paring function

$$
\begin{aligned}
& G_{\mathrm{d}-\operatorname{pair}}(\tau)=<0\left|d_{\sigma}(\tau) d_{\sigma^{\prime}}(0)\right| 3>\sim \tau^{-\alpha_{d}}, \\
& \text { or } \\
& G_{\mathrm{d}-\operatorname{pair}}(\omega) \sim \omega^{-1+\alpha_{d}},
\end{aligned}
$$

with the exponent

$$
\alpha_{d}=2 \delta_{0}^{* 2}-\delta_{x}^{* 2}\left(1-\delta_{\sigma, \sigma^{\prime}}\right) .
$$

The two-particle $d$-electron paring correlation function is given rise to

$$
<d_{\sigma} d_{\sigma^{\prime}}(\tau) d_{\sigma^{\prime}}^{\dagger} d_{\sigma}^{\dagger}(0)>\sim \tau^{-\alpha_{4}}
$$

with the exponent

$$
\alpha_{4}=-\left(\frac{\delta_{0}^{*}+\delta_{x}^{* 2}}{2}\right)^{2}
$$

We see that both $d$-electron pairing correlation functions are divergent in the low energy limit.

The $G_{2}$-irrelevant intermediate coupling mixed valence state is newly discovered at the finite $U$ model. The renormalization group equations yield an initially decreasing the paring charge fugacity $g_{2}$ and initially increasing the charge and spin fugacities $g_{1}$ and $g_{x}$. It can occur for which the spin and charge kinks are unbound while the paring charge kinks are bound. The two-body hybridization is irrelevant while the hybridization and exchange coupling are relevant. Therefore, the correlation functions such as $<0|R| 3>$ given by (4.8) and local pairing single- and two- $d$-electron correlation functions are still divergent. This gives a new set of local NFL fixed points.

\section{CONCLUSIONS}

We have constructed a general impurity model with two-body hybridization. Through comparing with the partition functions, via bosonization, we have found that this impurity problem is asymptotically equivalent to a six-component plasma of kinks with external fields. Hence, the renormalization group analysis can be systematically carried out in the framework of the AYHC poor man's scaling theory. We emphasize that it is important to introduce the two-body hybridization in the Hamiltonian. This ensures the transitivity of the 'dipole' operators and then the equivalence of the partition functions. Moreover, the renormalization group equations show that the flows excluding the two-body hybridization are not correct renormalization flows. In some phase of the phase diagram, $g_{2}$ is relevant, i.e., hybridizing electrons hop pairly onto and off the impurity orbital . This behavior of the electrons resembles the 'Cooper pair' in BCS theory. The property of the $g_{2}$-relevant phase is not clear yet. Perhaps, it relates to the superconduct phase in an impurity lattice model. In the phase diagram, there is a NFL phase which controlled by the fixed point behavior of the multi-channel X-ray edge problem. Around this phase we have six other phases called the $g_{2}$-relevant phase, the strong-coupling Kondo phase and four equivalent free orbital metallic phases. Hence, there is a FL-NFL phase transition in the system. There are six sequences of phase transitions from the NFL phase to 
the HT phase in which all fugacities are relevant. Each sequence has three critical points and four phases, which is same as the dimensions of the Lie algebra corresponding to the plasma 'charges'.

We have discussed various reduced models. For the spin symmetric model, the free orbital states do not exist because we can not freeze only one spin state in the impurity orbital and the free orbital condition can not be satisfied. For the PH symmetric model, we addressed that the PH symmetry of the common one-body hybridization term in the Hamiltonian forces $\epsilon_{d}=-U / 2$ by the consistency of the renormalization group equations and also decrease a critical point in the sequence of transitions, correspondingly reducing a dimension of the 'charges' Lie algebra.

We also discussed the fixed point behaviors in the mixed valence state regime. Except the X-ray edge type infrared orthogonality catastrophe in the screening channel, we also found the local NFL signal in the weak coupling and intermediate coupling mixed valence states.

\section{Acknowledgments}

One of us (Y. Y.) is grateful to Y. M. Li and N. d'Amberumenil for their co-operation in the earlier stage of this work and useful discussions. The authors thank Z. B. Su for discussions. This work was supported in part by NSF of China and Grant LWTZ-1298 of Chinese Academy of Science.

\section{APPENDIX A: LIE ALGEBRA AND ITS RELATION TO PHASE STRUCTURES}

In this appendix, we discuss some mathematical properties of the 'charges' $q_{\alpha \beta}^{a}$. The screening components, $q_{\alpha \beta}^{l}$, are trivial and we focus on the spin-components, $q_{\alpha \beta}^{\sigma}$. We define $q_{\alpha \beta}^{\gamma}$ are given by the spin-components for $\gamma=\sigma$ or vanish for $\gamma=0$ and 3. In terms of the transitivity (3.19) and the anti-symmetry of the 'charges', we immediately have $q_{\alpha \beta}^{\gamma}$ obey the Jacobi identities:

$$
\sum_{\gamma} q_{\alpha \beta}^{\gamma} q_{\gamma \delta}^{\lambda}+q_{\beta \delta}^{\gamma} q_{\gamma \alpha}^{\lambda}+q_{\delta \alpha}^{\gamma} q_{\gamma \beta}^{\lambda}=0
$$

which means that the 'charges' may be regarded as a set of structure constants of some Lie algebra. Denoting the generators of the Lie algebra by $T_{\alpha}$, then we have

$$
\begin{array}{r}
{\left[T_{0}, T_{\sigma}\right]=q_{0 \sigma}^{\sigma^{\prime}} T_{\sigma^{\prime}}, \quad\left[T_{\sigma}, T_{\sigma^{\prime}}\right]=q_{\sigma \sigma^{\prime}}^{\sigma^{\prime \prime}} T_{\sigma^{\prime \prime}},} \\
{\left[T_{\sigma}, T_{3}\right]=q_{\sigma 3}^{\sigma^{\prime}} T_{\sigma^{\prime}}, \quad\left[T_{0}, T_{3}\right]=q_{03}^{\sigma} T_{\sigma} .}
\end{array}
$$

The Lie algebra has the following properties:

(i) It is solvable. We call the Lie algebra $\mathcal{L}$. Equations (A2) imply that $[\mathcal{L}, \mathcal{L}] \subseteq \mathcal{L}_{1},\left[\mathcal{L}_{1}, \mathcal{L}_{1}\right] \subseteq \mathcal{L}_{2}$ and $\left[\mathcal{L}_{2}, \mathcal{L}_{2}\right]=0$, where $\mathcal{L}_{1}=\left\{T_{\sigma}\right\}$ and $\mathcal{L}_{2}=\left\{q_{\uparrow \downarrow}^{\sigma} T_{\sigma}\right\}$. This means that $\mathcal{L}$ is solvable. (ii) The Lie algebra is 4-dimensional, which is same as the dimensions of the impurity Hilbert space. The structure constants form the adjoint representation by $\left(T_{\alpha}\right)_{\beta \gamma}=q_{\alpha \beta}^{\gamma}$. For the general parameters $\delta_{0}, \tilde{\delta}_{0}, \delta_{x \sigma}$ and $\tilde{\delta}_{x \sigma}, a T_{0}+b T_{\uparrow}+c T_{\downarrow}+d T_{3}=0$ only if all coefficients $a, b, c$ and $d$ are zero.

(iii) In the infinite $U$ limit, the doubly-occupied state is completely suppressed in the discussion of the low energy behavior of the system. The physics has been discussed in [15] [18,25]. The impurity Hilbert space is 3 -dimensional, and correspondingly, the Lie algebra reduces to 3 -dimensional one: $\mathcal{L}=\left\{T_{0}, T_{\sigma}\right\}$.

(iv) In the PH symmetric model, $q_{0 \sigma}^{\gamma}=q_{-\sigma, 3}^{\gamma}$, which leads to the dimensions of the Lie algebra is reduced to 3 too. This consequence can be checked in the adjoint representation by the following linear transformation

$$
\begin{aligned}
& L_{0}=T_{0}+T_{3}+T_{\uparrow}+T_{\downarrow}, \\
& L_{1}=T_{0}-T_{3}+T_{\uparrow}+T_{\downarrow}, \\
& L_{2}=T_{0}-T_{3}+T_{\uparrow}-T_{\downarrow}, \\
& L_{3}=T_{0}+T_{3}-T_{\uparrow}-T_{\downarrow} .
\end{aligned}
$$

The transformation is a non-singular but one finds that $L_{3} \equiv 0$. This implies that the dimensions of the Lie algebra is 3 .

At the moment, it is not clear that what are the physical implication of the Lie algebra and the corresponding Lie group. We only know that there is a correspondence between the phase structures and the dimensions, $D$, of the Lie algebra: $D-1$ is equal to the largest numbers of the critical lines between the NFL phase and the HT phase.

\section{APPENDIX B: RENORMALIZATION GROUP EQUATIONS}

Our partition function (3.17) has arrived at a special form of the Cardy's general model with an external field added. Therefore, the renormalization group equations can be directly derived by using the AYHC poor man's scaling theory. The details of the derivation have been presented in [21] 24] 218,25]. Here we just address something in their derivation.

The renormalization group equations describe the flows of the dimensionless couplings, the fugacities $g_{\alpha \beta}$, the stiffness constants $K(\alpha, \beta)$, and the external fields $E_{\alpha}$, as the band width is reduced.

Firstly, when the cut-off $\tau_{0} \rightarrow \tau_{0}+d \tau_{0}$, to compensate the changes of $\tau_{0}$ 's power terms and external field terms in the partition function (3.17), the fugacities and the external fields change as follows:

$$
\begin{aligned}
\delta g_{\alpha_{i+1} \alpha_{i}} & =\left(d \ln \tau_{0}\right) g_{\alpha_{i+1} \alpha_{i}}\left(1+K\left(\alpha_{i+1}, \alpha_{i}\right)\right), \\
\delta E_{\alpha_{i}} & =E_{\alpha_{i}} d \ln \tau_{0} .
\end{aligned}
$$

The cut-off of integral limits also causes the variation of the logarithmic terms. After integrating out the $\tau_{i+1}$, 
we obtain the variation involving two such kinks $i, i+1$ and a third kink $j$ which is given by

$$
\begin{aligned}
& \left(d \ln \tau_{0}\right) g_{\alpha_{i} \alpha_{i+1}} g_{\alpha_{i+1} \alpha_{i+2}} \\
& \exp \left\{\tilde{K}\left(\alpha_{i}, \alpha_{i+1} ; \alpha_{j}, \alpha_{j+1}\right) \ln \frac{\tau_{j}-\tau_{i}}{\tau_{0}}\right. \\
& \left.+\tilde{K}\left(\alpha_{i+1}, \alpha_{i+2} ; \alpha_{j}, \alpha_{j+1}\right) \ln \frac{\tau_{j}-\tau_{i}-\tau_{0}}{\tau_{0}}\right\} \\
& \exp \left\{E_{\alpha_{i+2}}-E_{\alpha_{i+1}}\right\} \\
& \approx\left(d l n \tau_{0}\right) g_{\alpha_{i} \alpha_{i+1}} g_{\alpha_{i+1} \alpha_{i+2}} \\
& \exp \left\{\left(K\left(\alpha_{i}, \alpha_{j}\right)+K\left(\alpha_{i+2}, \alpha_{j+1}\right)\right.\right. \\
& \left.\left.-K\left(\alpha_{i}, \alpha_{j+1}\right)-K\left(\alpha_{i+2}, \alpha_{j}\right)\right) \ln \frac{\tau_{j}-\tau_{i}}{\tau_{0}}\right\} \\
& \exp E_{\alpha_{i+2}}-E_{\alpha_{i+1}} .
\end{aligned}
$$

The only approximation is $\tau_{j}-\tau_{i} \gg \tau_{0}$, which means the kinks are rare enough.

As long as $\alpha_{i} \neq \alpha_{i+2}$, this can be incorporated into a renormalization of $g_{\alpha_{i} \alpha_{i+1}}$, i.e.

$$
\delta g_{\alpha_{i} \alpha_{i+1}}=\left(d \ln \tau_{0}\right) \sum_{\alpha} g_{\alpha_{i} \alpha} g_{\alpha \alpha_{i+1}} e^{E_{\alpha_{i+1}}-E_{\alpha}},
$$

which together with (B.1) and $g_{\alpha \beta}=g_{\beta \alpha}$ leads to the renormalization flows of the fugacities in arbitrary external fields:

$$
\frac{d g_{\alpha \beta}}{d l n \tau_{0}}=(1+K(\alpha, \beta)) g_{\alpha \beta}+\sum_{\gamma} g_{\alpha \gamma} g_{\gamma \beta} e^{-E_{\gamma}+\frac{1}{2}\left(E_{\alpha}+E_{\beta}\right)} .
$$

However, for the neutral pair with $\alpha_{i}=\alpha_{i+2}$, the leading term of the left hand side of (B.2) is independent of the relative position of the kinks. It becomes:

$$
\left(d \ln \tau_{0}\right) g_{\alpha_{i} \alpha_{i+1}}^{2} \frac{\tau_{i+2}-\tau_{i-1}}{\tau_{0}} e^{E_{\alpha_{i}}-E_{\alpha_{i+1}}} .
$$

which leads to the renormalization of the external fields:

$$
\delta E_{\alpha}=-d \ln \tau_{0} \sum_{\beta} g_{\alpha \beta}^{2} e^{E_{\alpha}-E_{\beta}} .
$$

Taking into account $\sum E_{\alpha}=0$ and the renormalization of the free energy $F$, the renormalization flows of $E_{\alpha}$ and $F$ are

$$
\begin{aligned}
& \frac{d E_{\alpha}}{d l n \tau_{0}}=E_{\alpha}-\sum_{\gamma} g_{\alpha \gamma}^{2} e^{E_{\alpha}-E_{\gamma}}+\frac{1}{4} \sum_{\beta \gamma} g_{\beta \gamma}^{2} e^{E_{\beta}-E_{\gamma}}, \\
& \frac{d F \tau_{0}}{d \ln \tau_{0}}=-\frac{1}{4} \sum_{\alpha \beta} g_{\alpha \beta}^{2} e^{E_{\alpha}-E_{\beta}} .
\end{aligned}
$$

Then, we must consider the next term in the expansion of (B.2) as $\tau_{0} /\left(\tau_{j}-\tau_{i}\right)$, which is

$$
\begin{aligned}
& \left(d \ln \tau_{0}\right) g_{\alpha_{i} \alpha_{i+1}}^{2} \tilde{K}\left(\alpha_{i}, \alpha_{i+1} ; \alpha_{j}, \alpha_{j+1}\right)\left(\ln \left(\tau_{j}-\tau_{i+2}\right)\right. \\
& \left.-\ln \left(\tau_{j}-\tau_{i-1}\right)\right) e^{E_{\alpha_{i}}-E_{\alpha_{i+1}}}
\end{aligned}
$$

after integrated over the 'closed pair' 21] range $\tau_{i-1} \leq$ $\tau_{i} \leq \tau_{i+2}$. This can be interpreted as the renormalization of the stiffness constants:

$$
\begin{aligned}
\frac{d K(\alpha, \beta)}{d l n \tau_{0}}= & -\sum_{\gamma} g_{\alpha \gamma}^{2} e^{E_{\alpha}-E_{\gamma}} \\
& (K(\alpha, \beta)+K(\alpha, \gamma)-K(\beta, \gamma)) \\
- & \sum_{\gamma} g_{\beta \gamma}^{2} e^{E_{\beta}-E_{\gamma}} \\
& (K(\alpha, \beta)+K(\beta, \gamma)-K(\alpha, \gamma)) .
\end{aligned}
$$

Equations (B4)-( $(\mathrm{B} 6)$ and $(\mathrm{B} 8)$ are our renormalization group equations in the model. The relations $g_{\alpha, \alpha}=0$ and $K(\alpha, \alpha)=0$ and $\sum E_{\alpha}=0$ are preserved in the renormalization process.

[1] P. W. Anderson, Science 235, 1196(1987); Physica C 185-189, 11(1991); P. W. Anderson and R. Schrieffer, Phys. Today, June 1991, p.54.

[2] C. M. Varma, S. Schmitt-Rink and E. Abraham, Solid Stat. Commun. 62, 681(1987); C. M. Varma, P. B. Littlewood, S. Schmitt-Rink, E. Abraham and A. E. Ruckenstein, Phys. Rev. Lett. 63, 1996 (1989); A. E. Ruckenstein and C. M. Varma, Physica C 185-189, 134(1991).

[3] F. D. M. Haldane, J. Phys., C 14, 2585 (1981).

[4] See, e.g., R. Shankar, preprint cond-mat/9307009 and references therein.

[5] D. C. Mattis and E. Lieb, J. Math. Phys., 6,304(1965); A. Luther and I. Peschel, Phys. Rev. B 12, 3908 (1975); S. Coleman, Phys. Rev. D 11, 2088 (1975); S. Mandelstam, Phys. Rev. D 11, 3026 (1975).

[6] F. D. M. Haldane, Varenna Lecture, 1992 and Helv. Phys. Acta. 65, 152 (1992).

[7] A. Houghton and J. B. Marston, Phys. Rev. B 48, 7790 (1993).

[8] D. V. Khevshchenko, R. Hlubina and T. M. Rice, Phys. Rev. B 48 , 10766 (1993); A. H. Castro Neto and E. Fradkin, Urbara preprint, 1993; R. Hlubina, ETH preprint,1993; D. V. Khevshchenko, Princeton preprint, 1993.

[9] P. W. Anderson, Phys. Rev. Lett. 267, 3844 (1991).

[10] X. G. Wen, Phys. Rev. B 42, 6623 (1990); H. J. Schulz, Int. J. Mod. Phys. B 5, 57 (1991); M. Fabrizio, A. Parola and E. Tosatti, Phys. Rev. B 46, 3159 (1992); G. M. Zhang, S. P. Feng and L. Yu, to be published; A. Nersesyan, A. Luther and F. V. Kusmartev, Phys. Lett. A 176, 363 (1993); P. W. Anderson, JETP Lett. 58, 59 (1993); V. M. Yakovenko, ibid 56, 5101 (1992); D. V. Khevshchenko, preprint; Y. M. Li, Y. Yu, N. d'Amberumenil, L. Yu and Z. B. Su, Mod. Phys. Lett. B $\mathbf{8 , 7 4 9}$ (1994); and references therein. 
[11] J. Friedel, Can. J. Phys. 34, 1190 (1956); A. Blandin and J. Friedel, J. Phys. Radium 20, 160 (1959).

[12] P. W. Anderson, Phys. Rev. 124, 41(1961).

[13] K. G. Wilson, Rev. Mod. Phys. 47, 773(1975).

[14] P. Nozieres, J. Low Temp. Phys. 17, 31(1974).

[15] I. E. Perakis, C. M. Varma and A. E. Ruckenstein, Phys. Rev. Lett. 70, 3467 (1993).

[16] T. Giamarchi, C. M. Varma, A. E. Ruckenstein and P. Nozieres, Phys. Rev. Lett. 70, 3967 (1993).

[17] G. M. Zhang, L. Yu and Z. B. Su, Phys. Rev. B 49, 7759(1994).

[18] Q. M. Si and G. Kotliar, Phys. Rev. Lett. 70, 3143 (1993).

[19] P. B. Wiegmann and A. M. Finkel'stein, Sov. Phys. JETP 48, 102 (1978); P. Schlottmann, J. Phys. (Paris) 6, 1486(1978); Phys. Rev. B 45, 4815 (1982).

[20] J. Solyom, Adv. Phys. 28, 209 (1979); J. Phys. F 4, 2269 (1975); P. Nozieres, and A. Blandin, J. Phys. (Paris) 41, 193 (1980).

[21] P. W. Anderson, G. Yuval and D. R. Hamann, Phys. Rev. B1, 4464 (1970); P. W. Anderson, J. Phys. C3, 2436 (1970).

[22] G. Mahan, Phys. Rev. 153, 882 (1967); P. W. Anderson, Phys. Rev. Lett. 18, 1049 (1967); P. Nozieres and C. T. de Dominicis, Phys. Rev. 173, 1097 (1969).

[23] Yue Yu, Y.M. Li and N. d'Amberumenil, Phys. Rev. B 51, 16417 (1995).

[24] J. L. Cardy, J. Phys. A14, 1407 (1981); See also S. Chakravarty and J. Hirsch, Phys. Rev. B25, 3273 (1982).

[25] Q. Si and G. Kotliar, Phys. Rev. B48, 13881 (1993).

[26] V. J. Emery, in Highly Conducting One-dimensional Solids, eds. J. T. Devreese et al. (plenum, New York,1979); J. Solyom in 21. K. D. Schotte and U. Schotte, Phys. Rev. 182,479 (1969); K. D. Schotte, Z. Phys. 230, 99 (1970).

[27] C. Sire, C. M. Varma, A. E. Ruckenstein and T. Giamarchi, Phys. Rev. Lett.72,2478 (1994).

[28] G. M. Zhang and L. Yu, Phys. Rev. Lett. 72, 2474 (1994).

[29] In 15], this term was discussed as the leading term of the fixed point Hamiltonian in the second order.

[30] F. M. D. Haldane, Phys. Rev. Lett. 40, 416 (1978); J. Phys. C 11, 5015 (1978).

[31] In the language of conformal field theory, $O(\alpha, \beta)$ is called vertex operator; $q_{\alpha \beta}^{a}$ is still called charge; and $K(\alpha, \beta)$ is the conformal weight of the vertex operator $O(\alpha, \beta)$.

[32] See P. B. Wiegmann and A. M. Finkel'stein in [19].

[33] See, for example, P. Nozieres and C. T. de Dominicis in 22]; Also see K. D. Schotte and U. Schotte in 26. Recent application, see 15 . 


\section{Figure Captions}

Fig. 1. The phase structure of the spin symmetric model. (a) $K_{0 \sigma}<K_{\sigma 3}, K_{03}, K_{\uparrow \downarrow}$. (b) $K_{\sigma 3}<$ $K_{0 \sigma}, K_{03}, K_{\uparrow \downarrow}$. (c) $K_{\uparrow \downarrow}<K_{0 \sigma}<K_{\sigma 3}, K_{03}$. (d) $K_{\uparrow \downarrow}<K_{\sigma}<K_{0 \sigma}, K_{03}$. (e) $K_{03}<K_{0 \sigma}, K_{\sigma 3}, K_{\uparrow \downarrow}$.

Fig. 2. The phase structure of the PH symmetric model.

(a) $K_{0 \uparrow}<K_{0 \downarrow}, K_{\uparrow \downarrow}, K_{03}$; (b) $K_{0 \downarrow}<K_{0 \uparrow}, K_{\uparrow \downarrow}, K_{03}$; (c) $K_{\uparrow \downarrow}<K_{0 \sigma}<K_{03}$; (d) $K_{03}<K_{0 \sigma}<K_{\uparrow \downarrow}$ or $K_{03}<K_{0 \sigma}<K_{\uparrow \downarrow}<K_{0,-\sigma}$.
Fig. 3. The phase structure of the spin and $\mathrm{PH}$ symmetric model. (a) $K_{0 \sigma}=K_{\uparrow \downarrow}=K_{03}$; (b) $K_{\uparrow \downarrow}<$ $K_{0 \sigma}<K_{03}$; (c) $K_{03}<K_{0 \sigma}<K_{\uparrow \downarrow}$. (d) The phase diagram in $\gamma_{0}-\gamma_{x}$ space, where $\gamma_{0}=\left(1-\delta_{x} / \pi-\right.$ $\left.\delta_{0} / \pi\right)^{2}+\left(\delta_{0} / \pi\right)^{2}+\sum_{l}\left(\delta_{l} / \pi\right)^{2}$ and $\gamma_{x}=\left(1-\delta_{x} / \pi\right)^{2}$. (e) The real phase diagram in $\gamma_{0}-\gamma_{x}$ space. The thick lines are phase boundaries. The thin lines divide the Fermi liquid phase into the different regions characterized by different behaviors at finite temperature. 
(a)
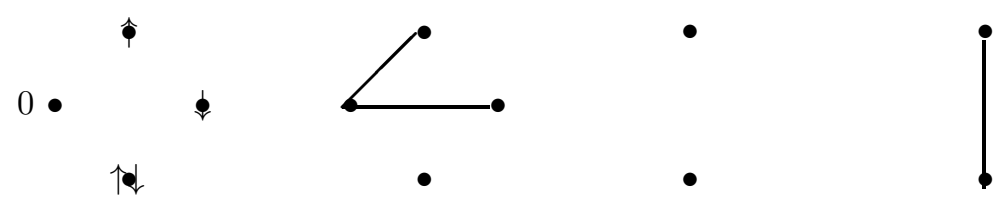

(b)
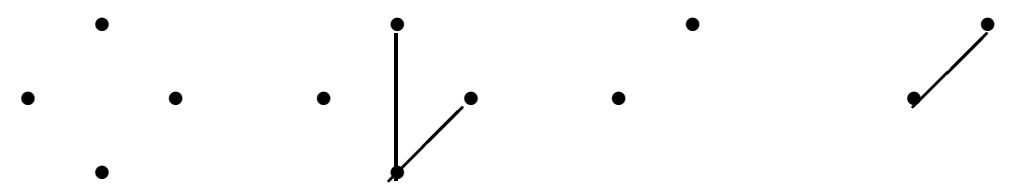

(c)

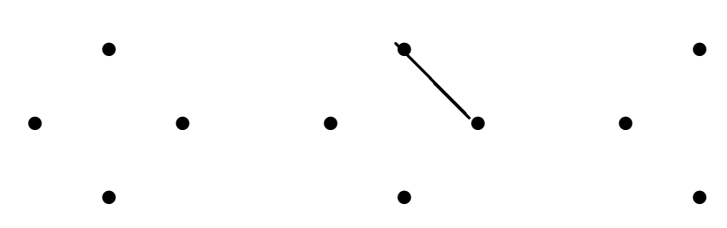

(d)
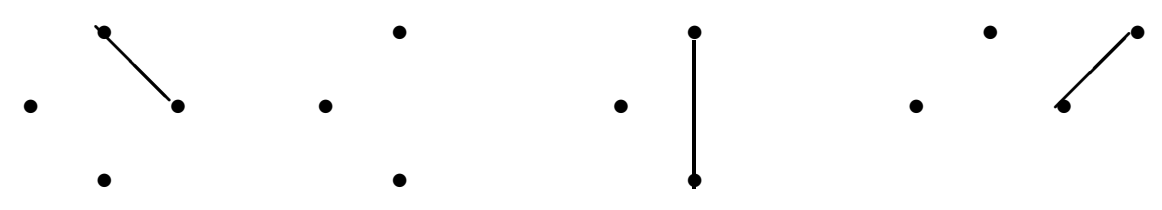

(e)
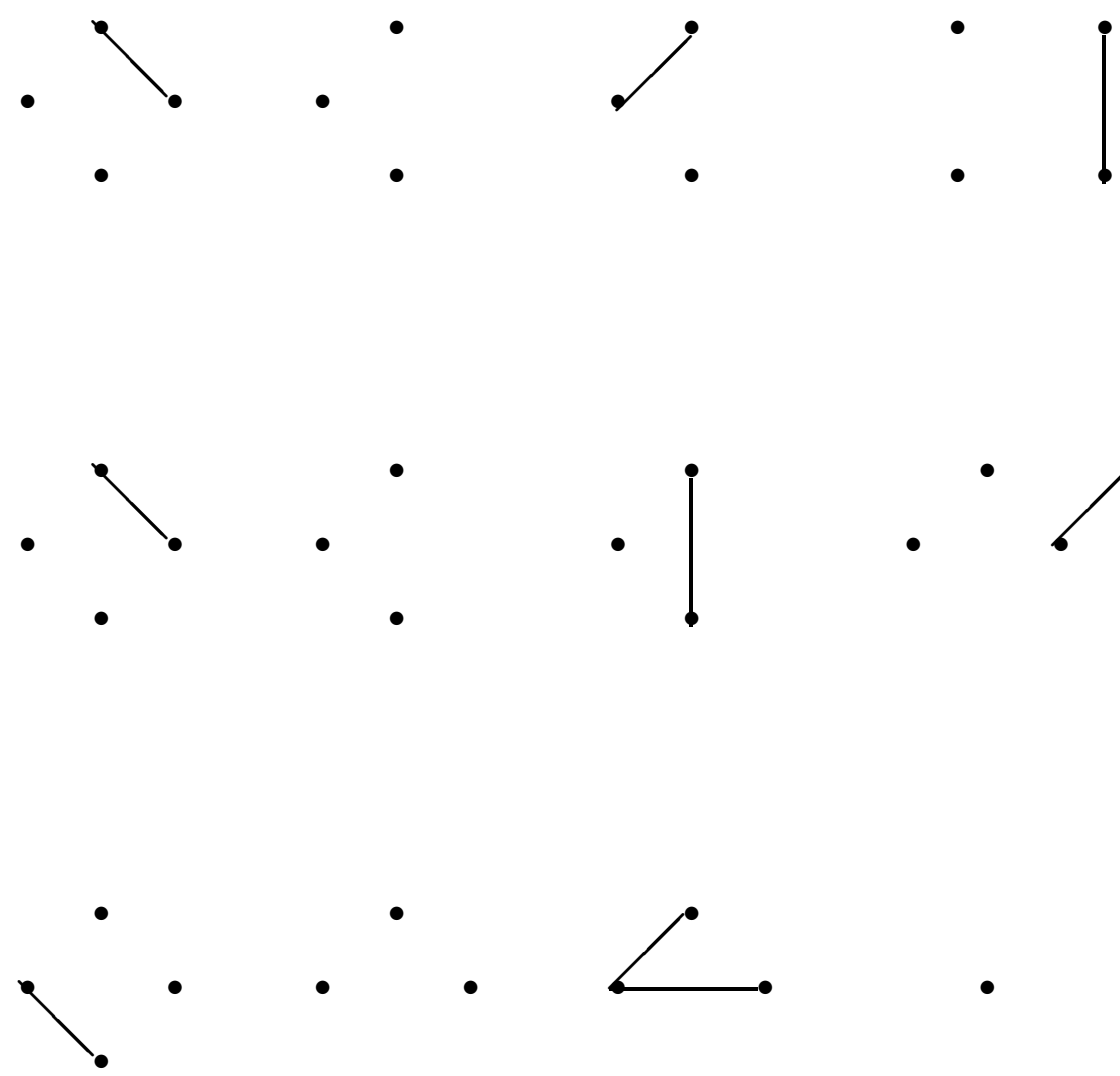

FIGURE 1 
(a)
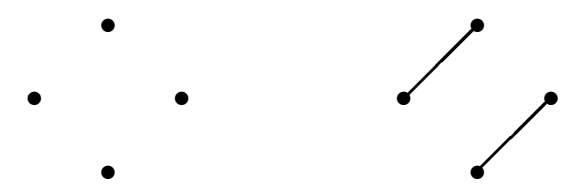

.
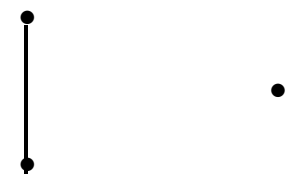

-

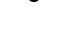

(b)
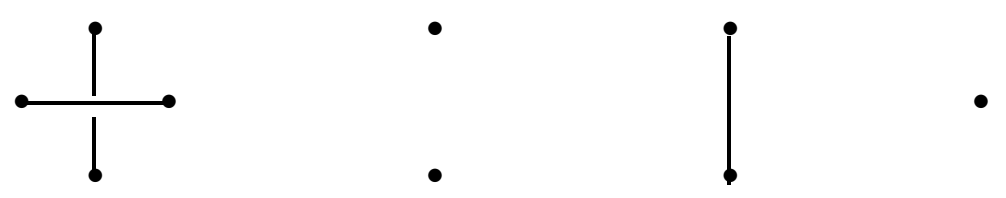

(c)
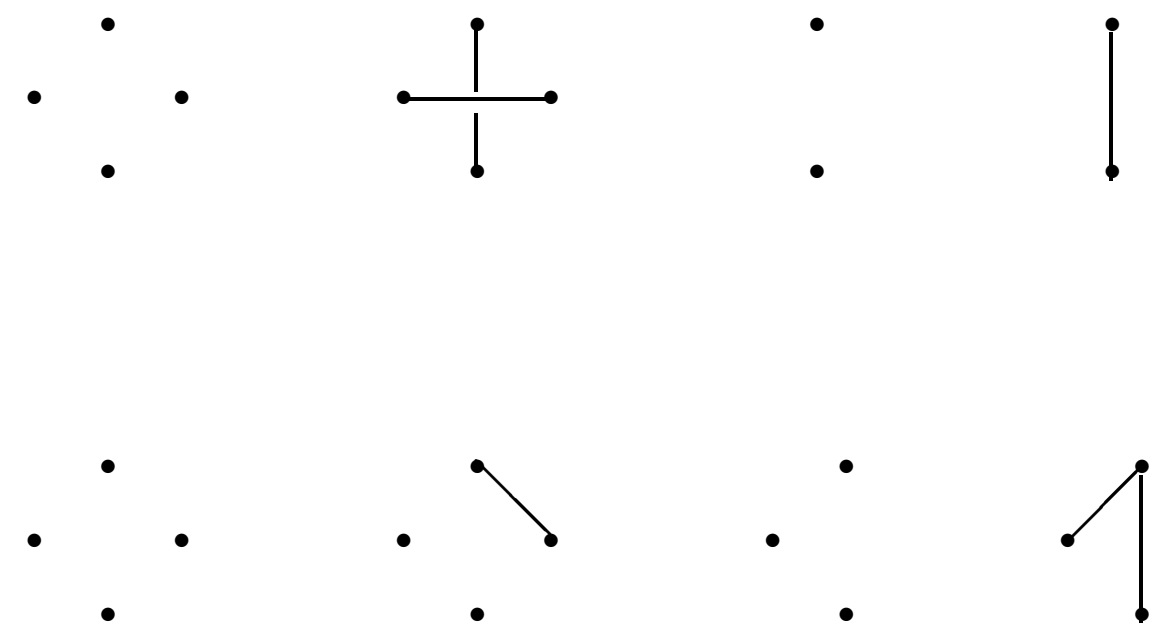

(d)
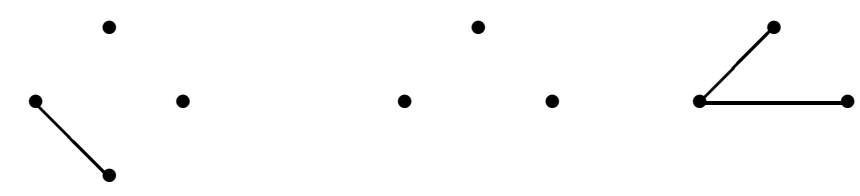

•

FIGURE 2 
(a)
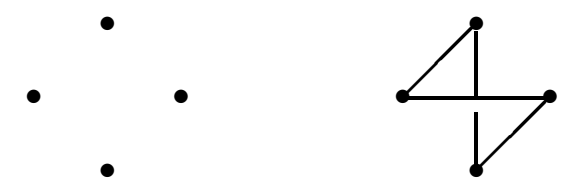

(b)

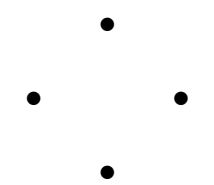

(c)
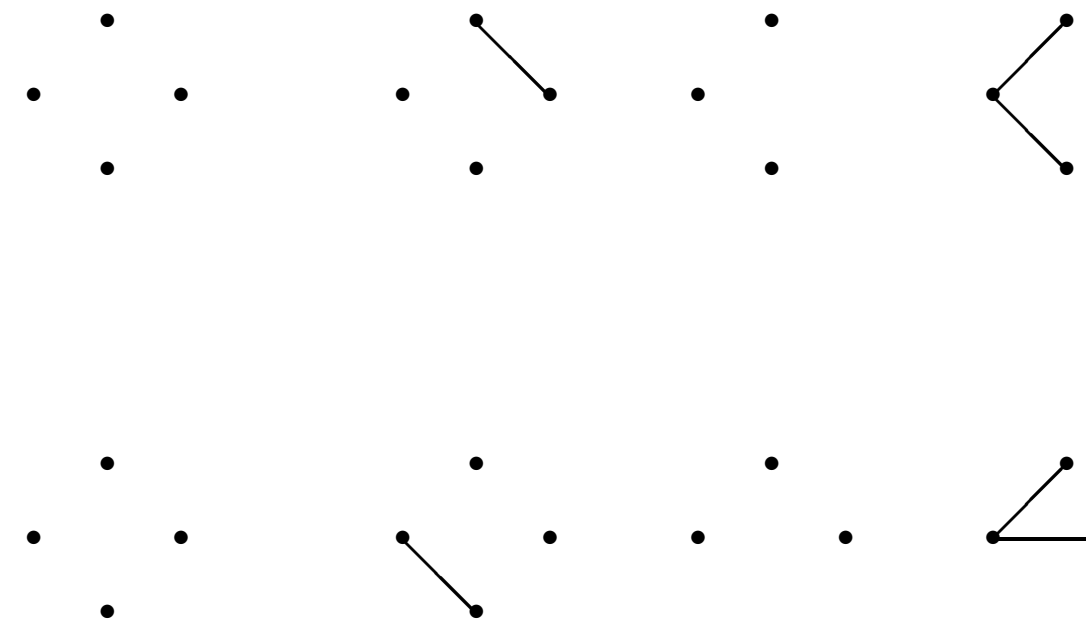

FIGURE 3(a)(b)(c) 
$\gamma_{x}$

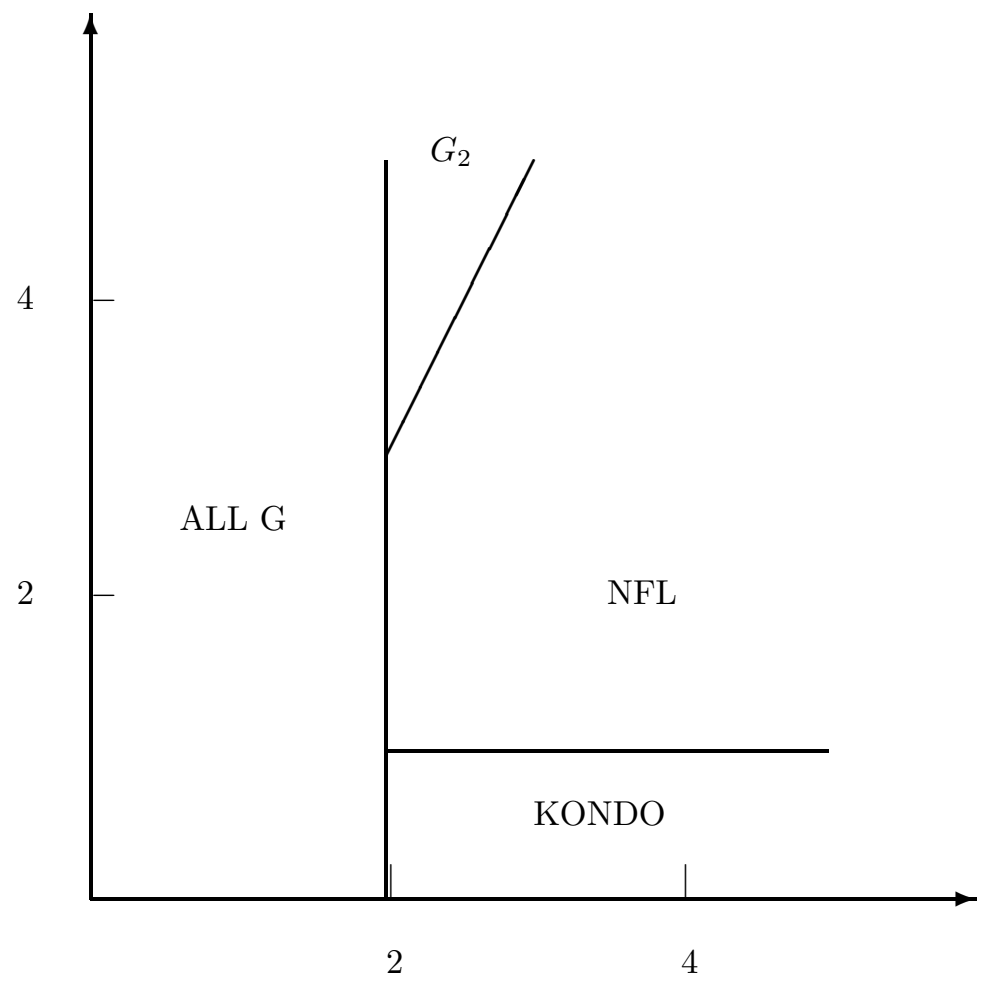

$\gamma_{0}$

FIGURE 3(d) 


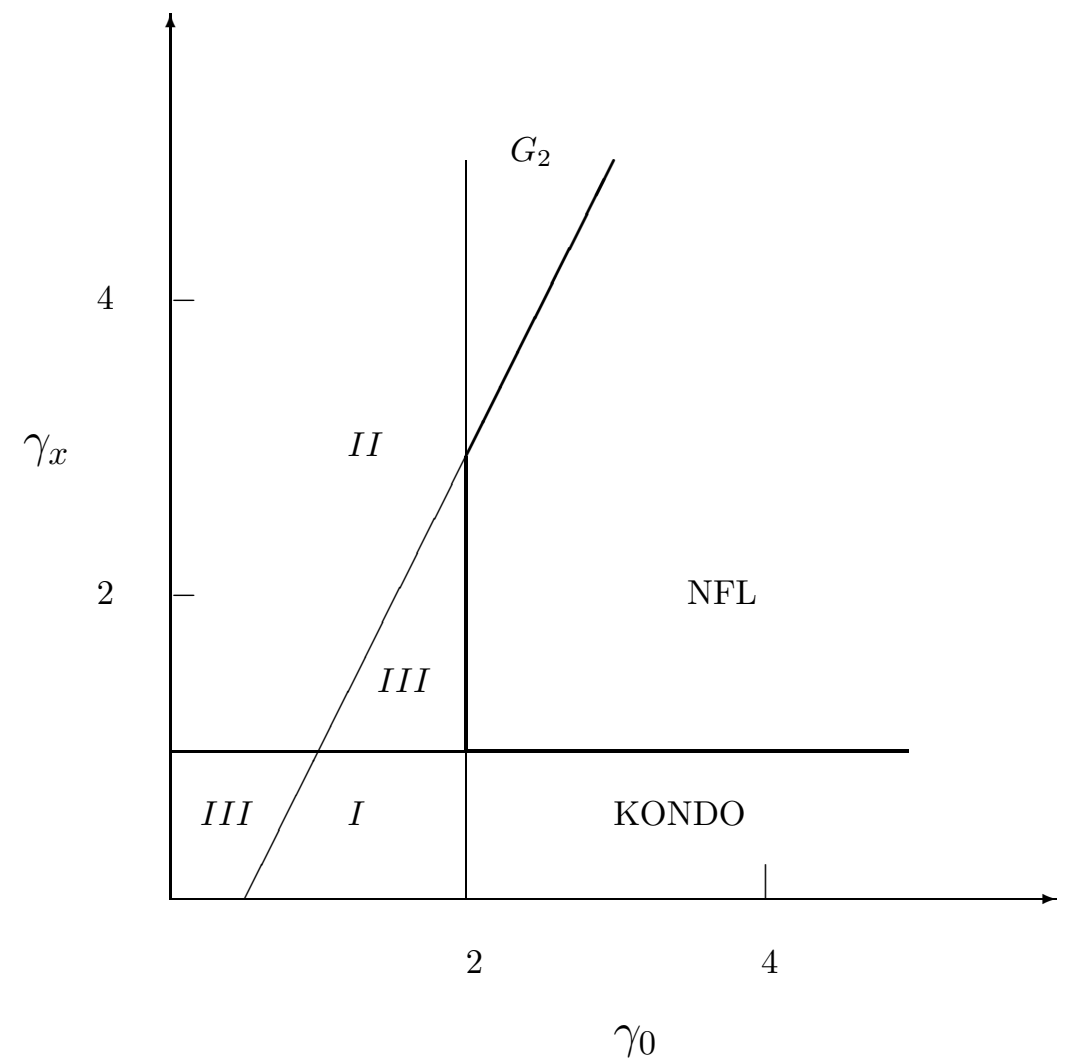

FIGURE 3(e) 